\title{
A tomografia computadorizada melhora a reprodutibilidade na classificação das fraturas transtrocanterianas?*
}

\section{Does Computed Tomography improve Reproducibility in the Classification of Transtrochanteric Fractures?}

\author{
Murilo Alexandre $^{1}$ Giancarlo Cavalli Polesello ${ }^{1,2}$ Edio Cavassani Neto ${ }^{1}$ \\ Nayra Deise dos Anjos Rabelo ${ }^{1}$ Marcelo Cavalheiro de Queiroz ${ }^{1,2}$ Walter Ricioli Junior ${ }^{1,2}$ \\ ${ }^{1}$ Grupo de Quadril, Departamento de ortopedia e Traumatologia, \\ Faculdade de Ciências Médicas da Santa Casa de São Paulo (FCMSCSP), \\ Irmandade Santa Casa de Misericórdia, São Paulo, SP, Brasil \\ Endereço para correspondência Giancarlo Cavalli Polesello, MD, PhD, \\ Rua Dr. Cesário Mota Júnior, 112, Bairro Vila Buarque, São Paulo, \\ SP, 01221-020, Brasil (e-mail: dot.quadril@hotmail.com).
}

2 Faculdade de Ciências Médicas, Santa Casa de São Paulo, São Paulo, SP, Brasil

Rev Bras Ortop 2019;54:361-367.

\section{Resumo}

Palavras-chave

- fraturas do quadril/ classificação

- estudos de validação

- tomografia computadorizada por raios $x$

- radiografia
Com o envelhecimento populacional, houve um aumento significante da prevalência das fraturas do quadril, com alto índice de mortalidade, de sequelas, e alto custo. Compreender o perfil da fratura e classificá-la de forma correta é fundamental para definir o tratamento adequado. Diversas classificações radiográficas foram desenvolvidas para as fraturas transtrocanterianas, tais como as de Tronzo, de Evans-Jensen, de Boyd-Griffin e AO, porém sua reprodutibilidade nem sempr é satisfatória. O presente trabalho objetivou analisar se o acréscimo da tomografia computadorizada (TC)implica em maior reprodutibilidade do que a radiografia simples na classificação das fraturas transtrocanterianas e se esta é melhor para a identificação do traço de fratura. Foi realizada uma pesquisa nas bases de dados PubMed, Lilacs, Scielo e Cochrane entre julho de 2016 e junho de 2017, limitada aos últimos 15 anos. Todos os trabalhos retrospectivos, prospectivos e revisões sistemáticas publicados na língua inglesa, com avaliação de homens e/ou de mulheres, foram considerados para a revisão. Foram excluídos relatos de casos, estudos que avaliaram de forma isolada a TC ou radiografias e estudos duplicados. A pesquisa apresentou 112 artigos, dos quais 5 preencheram os critérios propostos. A reprodutibilidade para a classificação das fraturas transtrocanterianas apresentou resultados variáveis e influenciados por fatores como o tipo de classificação, o uso da classificação simplificada ou completa, a especialidade do avaliador, a experiência e a metodologia proposta pelos trabalhos. Há indícios de que há algum benefício para o uso da TC, sobretudo para fraturas consideradas instáveis,

* Trabalho feito no Grupo de Quadril, Departamento de Ortopedia e Traumatologia, Irmandade da Santa Casa de Misericórdia de São Paulo, São Paulo, SP, Brasil.

(D) Giancarlo Cavalli Polesello's ORCID is https://orcid.org/0000-00029524-8871.

recebido

06 de Março de 2018

aceito

30 de Outubro de 2018
DOI https://doi.org/

10.1055/s-0039-1693045. ISSN 0102-3616.
Copyright $\odot 2019$ by Sociedade Brasileira License terms de Ortopedia e Traumatologia. Published by Thieme Revnter Publicações Ltda, Rio de Janeiro, Brazil 


\section{Abstract}

\author{
Keywords \\ - hip fractures/ \\ classification \\ - validation studies \\ - tomography x-ray \\ computed \\ - radiography
}

porém sua utilização como ferramenta para garantir uma melhor reprodutibilidade (intra- e interobservador) ainda permanece controversa e carece de mais estudos.

With the aging of the population, there was a significant increase in the prevalence of hip fractures, with high mortality rates, sequelae and expenses. Understanding the fracture profile and classifying it correctly is critical to define the appropriate treatment. Several radiographic classifications have been developed for transtrochanteric fractures, such as Tronzo, Evans-Jensen, AO and Boyd-Griffin, but their reproducibility is not always satisfactory. The present review aimed to elucidate whether the addition of computed tomography (CT) implies a greater reproducibility than simple radiography in the classification of transtrochanteric fractures, and whether this is a better examination to identify the fracture trait. A search was conducted in the PubMed, Lilacs, Scielo and Cochrane databases between July 2016 and June 2017, limited to the last 15 years. All retrospective, prospective and systematic reviews articles published in the English language, with evaluation of men and/or women, were considered for review. We have excluded case reports, studies that evaluated tomography or radiographs in isolation, and duplicate studies. The research presented 112 articles, of which 5 contemplated the proposed criteria. Reproducibility for the classification of transtrochanteric fractures presented variable results and was influenced by factors such as the type of classification, the use of the simplified or complete classification, the specialty of the evaluator, his experience, and the methodology proposed by the works. There are indications that there is benefit for the use of $C T$, especially for fractures considered unstable, but its use as a tool to ensure better reproducibility (intraand interobserver) remains controversial and needs further studies.

\section{Introdução}

Fraturas transtrocanterianas são aquelas compreendidas entre a base do colo femoral até $2,5 \mathrm{~cm}$ distal ao trocanter menor. ${ }^{1}$ Embora possam ocorrer em jovens após trauma de alta energia, os idosos vítimas de queda ao solo são os mais acometidos. $^{2}$ Com o envelhecimento populacional, a prevalência de osteoporose e de fraturas do quadril sofreu um aumento significativo, elevando os índices de mortalidade, as sequelas e os gastos decorrentes, ${ }^{3-7}$ quando comparados à prevalência e aos índices listados de outras fraturas. ${ }^{3,8-10}$

O diagnóstico é baseado na anamnese e na avaliação clínica, no qual um traumatismo de baixa energia em individuo idoso acarreta incapacidade funcional, encurtamento e atitude rotação externa do membro inferior. ${ }^{11}$ Pela avaliação radiográfica, com análise de imagens no plano anteroposterior da bacia (com rotação interna e tração dos membros inferiores) e incidência em perfil do quadril, confirma-se o diagnóstico bem como a característica da fratura. ${ }^{11}$

A boa compreensão do tipo de fratura e sua classificação correta são fundamentais para definir o tipo de osteossíntese mais adequado. ${ }^{12}$ Dessa forma, o sistema de classificação, para ser apropriado na prática clínica diária, deve ser simples, de fácil aplicação, e apresentar boa reprodutibilidade tanto intra- quanto interobservador. ${ }^{13}$ Para atingir este objetivo, diversas classificações radiográficas foram desenvolvidas como as de Boyd et al, ${ }^{14}$ de Tronzo, ${ }^{8}$ de Jensen, ${ }^{15}$ de Evans, ${ }^{16}$ e a classificação AO. ${ }^{1}$ Entretanto, em alguns casos, ocorrem divergências classificatórias que prejudicam a reprodutibilidade intra- e interobservador. ${ }^{17-19}$ Para tais casos, pode ser necessário utilizar métodos diagnósticos que possibilitem uma melhor identificação e concordância, a fim de permitir uma boa escolha do tratamento. ${ }^{2,17,18,20,21}$

A tomografia computadorizada (TC) é uma técnica radiológica mais avançada, gerando imagens mais detalhadas e completas, muito úteis para a avaliação de fraturas complexas e articulares, tais como do pilão tibial, ${ }^{22,23}$ do planalto tibial, ${ }^{24,25}$ do úmero distal ${ }^{26}$ e do calcâneo. ${ }^{27}$ Seguindo este princípio, foi proposta sua utilização em fraturas transtrocanterianas com o uso de classificação tomográfica, como sugerida por Nakano. ${ }^{28}$

A disponibilidade e o uso deste exame suscitou a possibilidade de a TC apresentar melhor reprodutibilidade intra- e interobservador, bem como maior precisão diagnóstica para o uso de rotina. ${ }^{20,29-31}$ Porém, ainda existem controvérsias na literatura. Há trabalhos que apresentam a técnica como dispensável e outros como superior quando comparada às radiografias. ${ }^{28,32,33}$

Diante disso, avaliamos através de uma revisão sistemática se o acréscimo da TC apresenta reprodutibilidade intrae/ou interobservador superior à avaliação radiográfica simples isoladamente na classificação das fraturas.

\section{Materiais e Métodos}

Foi realizada pesquisa nas bases de dados PubMed, Lilacs, Scielo e Cochrane entre julho de 2016 e junho de 2017 , limitada aos últimos 15 anos. Utilizaram-se os descritores: (intertrochanteric OR hip) AND fracture AND (classification OR Tronzo OR AO OR Evans Jensen OR Boyd Griffin) AND (reproducibility 
OR validation studies OR reliability) AND (tomography OR CT) AND ( $x$-ray OR radiography). Na pesquisa inicial, todos os artigos que apresentaram dois ou mais termos no título e no resumo foram incluídos para a revisão.

Após a revisão dos artigos, foi realizado um cruzamento das referências para assegurar que todos os estudos em potencial fossem incluídos. Todos os trabalhos retrospectivos, prospectivos e revisões sistemáticas de língua inglesa, com avaliação de homens e/ou de mulheres, que compararam a reprodutibilidade da análise radiográfica e tomográfica das classificações de fraturas transtrocanterianas foram considerados para a revisão. Foram excluídos relatos de casos, estudos que avaliavam de forma isolada a tomografia ou radiografias, estudos duplicados, e estudos diagnósticos de fraturas ocultas ou por estresse.

Dos estudos incluídos, foram extraídos o nome do primeiro autor, o ano de publicação, o país, o desenho do estudo, o objetivo do estudo, o tamanho da amostra, o número de avaliadores, bem como suas especialidades, a classificação utilizada, o método de análise, e os resultados. Estes dados foram expressos em forma de tabela através do Microsoft Excel, versão 2016 (Microsoft Corporation, Redmond, WA, EUA) e analisados descritivamente. Foi seguida a recomendação Principais Itens para Relatar Revi- sões Sistemáticas e Meta-análises (PRISMA, na sigla em inglês) para a confecção da revisão sistemática. ${ }^{34}$

A reprodutibilidade dos estudos foi interpretada de acordo com o valor do coeficiente kappa, baseado nas diretrizes propostas por Landis et al: 0,00 a 0,20: ruim, 0,21 a 0,40: razoável; 0,41 a 0,60 moderada; 0,61 a 0,80 forte; 0,81 a 1,00 : excelente. ${ }^{35}$

\section{Resultados}

Inicialmente, a pesquisa apresentou 112 artigos, e após a aplicação dos critérios de inclusão e exclusão, foram obtidos 5 que satisfaziam os critérios propostos. Publicados entre os anos de 2003 e 2017, eles apresentam um mínimo de 30 e um máximo de 110 pacientes, com média de idade variando entre 59 e 85 anos. Devido ao número restrito de artigos, não foram considerados conflitos de interesses ou nível de evidência como critérios de exclusão (-Fig. 1).

Todos os trabalhos utilizaram a classificação AO, dois deles utilizaram a classificação Evans-Jensen, e apenas um a de Boyd-Griffin (conforme evidenciado na tabela 1).

Três artigos concluíram que o uso de TC apresenta benefícios para a correta classificação, com boa reprodutibilidade das fraturas transtrocanterianas, especialmente na

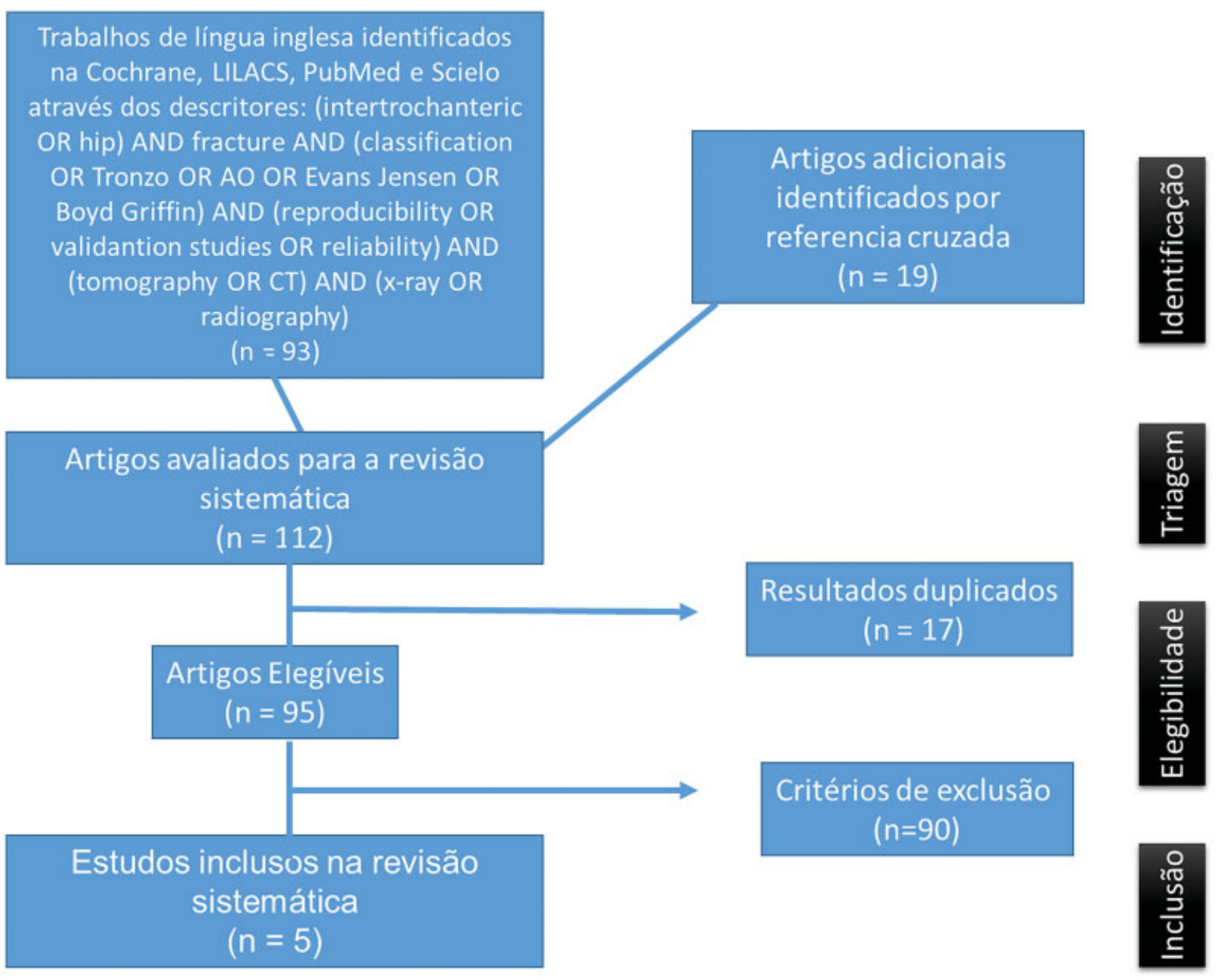

Fig. 1 Protocolo Principais Itens para Relatar Revisões Sistemáticas e Meta-análises (PRISMA, na sigla em inglês) para revisão de literatura. 
Tabela 1 Resultado da revisão sistemática

\begin{tabular}{|l|l|l|l|l|l|}
\hline Artigos & Amostra & Avaliadores & Classificação & Índice & Resultado \\
\hline $\begin{array}{l}\text { Chapman CB } \\
\text { et al, 2003. }\end{array}$ & 61 pacientes & $\begin{array}{l}3 \text { ortopedistas e } \\
2 \text { radiologistas }\end{array}$ & AO e Evans-Jensen & kappa & $\begin{array}{l}\text { Sem Alteração } \\
\text { da Reprodutibilidade }\end{array}$ \\
\hline $\begin{array}{l}\text { Cavaignac E } \\
\text { et al, 2013. }\end{array}$ & 53 pacientes & $\begin{array}{l}1 \text { residente de radiologia e } \\
1 \text { radiologista }\end{array}$ & $\mathrm{AO}^{1}$ e Evans-Jensen & kappa & $\begin{array}{l}\text { Sem Alteração da } \\
\text { Reprodutibilidade }\end{array}$ \\
\hline $\begin{array}{l}\text { Mihir Thanvi } \\
\text { et al, 2013. }\end{array}$ & 31 pacientes & $\begin{array}{l}2 \text { ortopedistas e2 } \\
\text { radiologistas }\end{array}$ & $\mathrm{AO}^{1}$ e Boyd Griffing & kappa & $\begin{array}{l}\text { Melhor } \\
\text { Reprodutibilidade }\end{array}$ \\
\hline $\begin{array}{l}\text { Isida } \text { R } \\
\text { et al, 2015. }\end{array}$ & 110 pacientes & $\begin{array}{l}1 \text { ortopedista e } \\
1 \text { radiologista }\end{array}$ & $\mathrm{AO}^{1}$ & kappa & $\begin{array}{l}\text { Melhor } \\
\text { Reprodutibilidade }\end{array}$ \\
\hline $\begin{array}{l}\text { Van Embden D } \\
\text { et al, 2016. }\end{array}$ & 30 pacientes & $\begin{array}{l}4 \text { ortopedistas, } \\
5 \text { radiologistas e } \\
2 \text { residentes de ortopedia }\end{array}$ & $\mathrm{AO}^{2}$ & kappa & $\begin{array}{l}\text { Melhor } \\
\text { Reprodutibilidade }\end{array}$ \\
\hline
\end{tabular}

1. Classificação AO completa (com subgrupos)

2. Classificação AO simplificada (sem subgrupos)

identificação de cominuição e de traços instáveis. ${ }^{29,30,33} \mathrm{Em}$ contrapartida, dois artigos indicaram que a TC não apresenta melhor reprodutibilidade da classificação quando comparada à radiografia simples ${ }^{20,33}$ ( $\mathbf{-}$ Tabela $\mathbf{1}$ ).

\section{Discussão}

A reprodutibilidade para a classificação das fraturas transtrocanterianas apresentou resultados variáveis e influenciados por fatores diversos, como o tipo de classificação, o número da amostra, o uso da classificação AO de forma simplificada ou completa, a especialidade dos avaliadores (radiologista versus ortopedista), a experiência dos avaliadores (staff versus residente) e a metodologia proposta por cada trabalho. Sendo assim, a discussão foi desenvolvida baseada em perguntas sobre os diversos fatores que poderiam influenciar tais resultados.

\section{Qual classificação é mais reprodutível?}

Verificou-se maior reprodutibilidade para a classificação AO simplificada quando comparada a outras classificações, como a AO completa e seus subgrupos, a Evans-Jensen e a Boyd-Griffin, ${ }^{20,29,33}$ tanto por TC quanto por radiografia. Provavelmente, tais resultados devam-se ao menor número de informações presentes na classificação AO simplificada, o que tornaria tanto sua compreensão quanto memorização mais fáceis quando comparada à classificação completa.

Ao analisar a TC, Ito et $\mathrm{al}^{36}$ evidenciaram concordância de $75 \%$ com a classificação AO completa e de $65,9 \%$ com a classificação de Evans-Jensen. Van Embden D. et al..$^{33}$ não apresentaram maior reprodutibilidade para a classificação AO simplificada ao acrescentar a TC ao estudo radiográfico, demonstrando valores kappa de 0,70 (forte) sem o uso da TC e de 0,68 (forte) com a TC. Cavaignac E. et al. ${ }^{20}$ apresentaram reprodutibilidade razoável com a adição da TC na classificação AO completa (de 0,28 para 0,33 ), enquanto que para a classificação de Evans-Jensen, a reprodutibilidade apenas com radiografia foi moderada $(0,50)$, e com radiografia associada à TC foi razoável $(0,35)$.
Isida R. et al. ${ }^{30}$ encontraram forte reprodutibilidade utilizando a TC com a classificação AO completa (kappa de 0,78), com valor preditivo positivo de $100 \%$, preditivo negativo de $79 \%$, e especificidade de $95 \%$ para a presença de cominuição. Quando analisados apenas por radiografias, observaram $48 \%$ de sensibilidade e $29 \%$ de valor preditivo negativo.

\section{A padronização da técnica radiográfica e tomográfica pode influenciar a reprodutibilidade?}

Apesar de a TC ter sua importância bem documentada em fraturas complexas e intra-articulares, ${ }^{22-27}$ é possível que nas fraturas transtrocanterianas os resultados tenham sido influenciados pelas divergências entre as técnicas radiológicas utilizadas.

Cavaignac E. et al. ${ }^{20}$ e Shen et al ${ }^{31}$ analisaram radiografias anteroposterior (AP) e de perfil (P) do quadril em apenas $56 \mathrm{e}$ $68 \%$ dos casos, respectivamente. Método inadequado, pois Koval et $\mathrm{al}^{11}$ demonstraram previamente que a avaliação radiográfica para as fraturas transtrocanterianas deve seguir um padrão no qual as duas incidências radiográficas (AP e P) devem ser realizadas em todos os casos. É inegável a existência de limitações para a obtenção de exames radiográficos, tais como sua realização nas salas de trauma, dor e dificuldade de posicionamento, porém a criação de protocolos para a padronização dos exames é de suma importância. ${ }^{12}$

O mesmo ocorre com a TC, apesar de uma certa padronização na realização destes exames. Observou-se viés relacionado a esta técnica no estudo de Cavaignac E. et al., ${ }^{20}$ no qual utilizaram apenas cortes axiais da TC, enquanto que Van Embden D. et al..$^{33}$ utilizam três planos tomográficos (coronal, sagital e axial), porém sem reconstrução 3D. Além disso, outro fator associado é a falta de especificação da técnica nos demais trabalhos analisados, tais como o número de radiografias, os planos utilizados nas radiografias e na TC, o posicionamento do paciente e a utilização ou não de modelos de reconstrução 3D.

Pode-se inferir que a limitação dos exames e a realização inadequada dos mesmos pode ter dificultado a avaliação e gerado viés nos resultados de reprodutibilidade encontrados. 


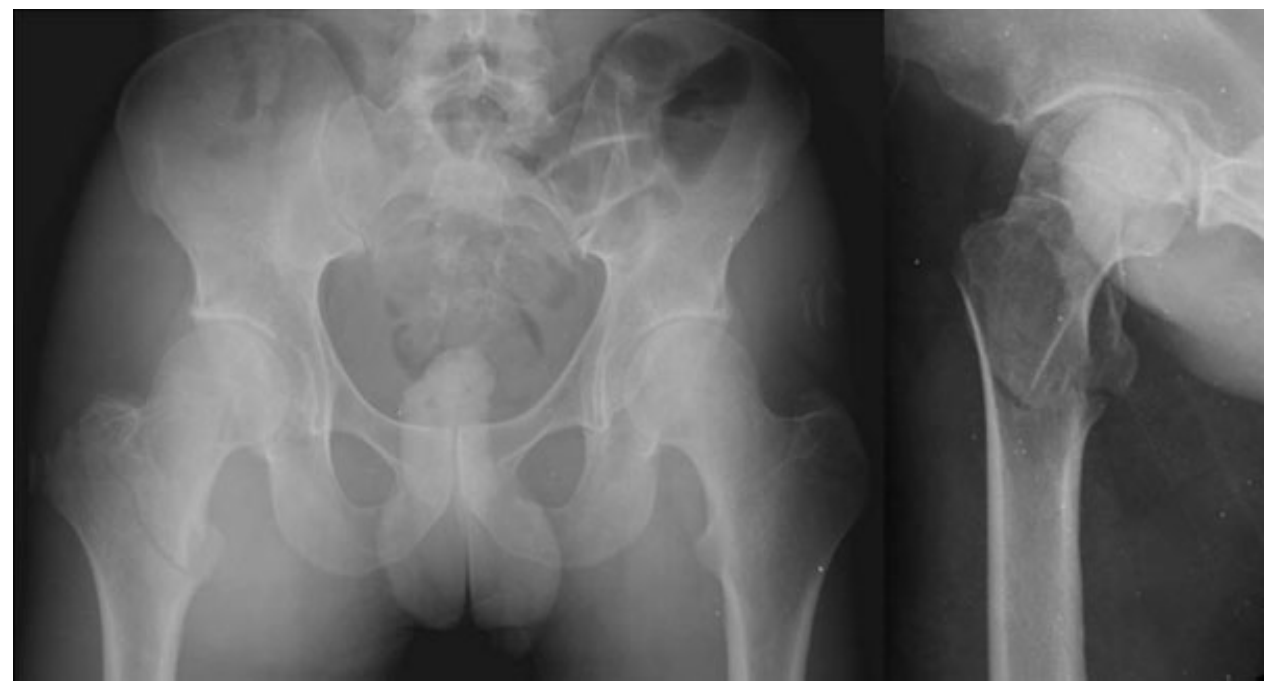

Fig. 2 Radiografias anteroposterior da bacia e perfil coxofemoral; classificação AO 31 A1.

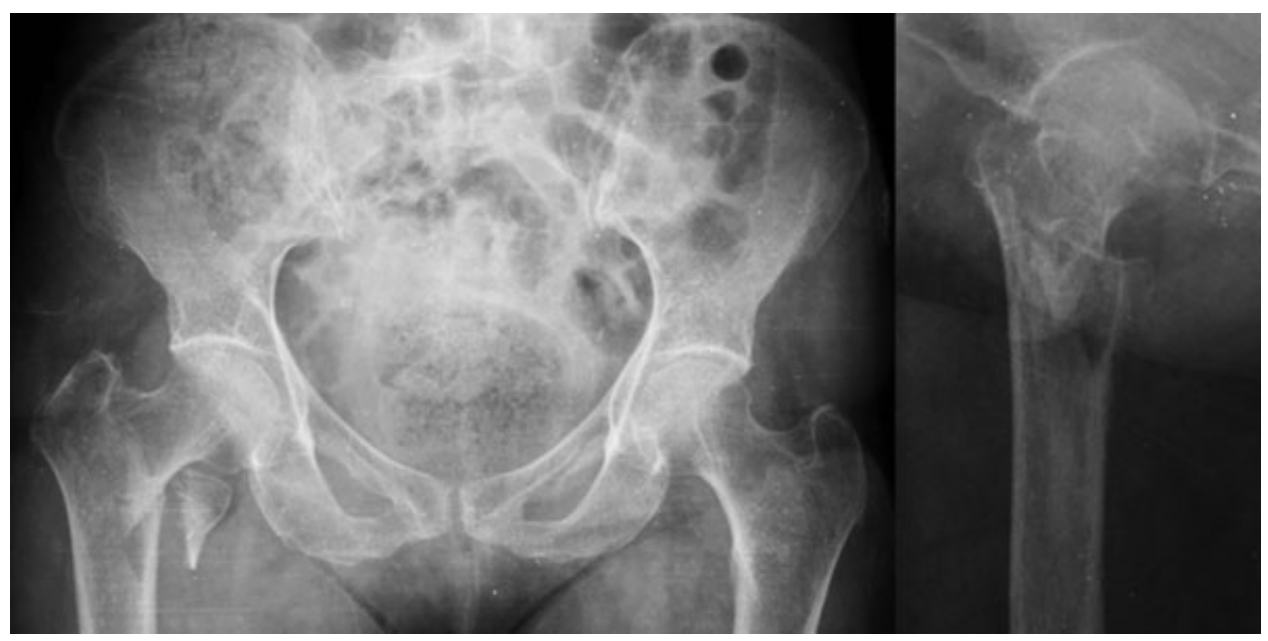

Fig. 3 Radiografias anteroposterior da bacia e perfil coxofemoral; classificação AO 31 A2.

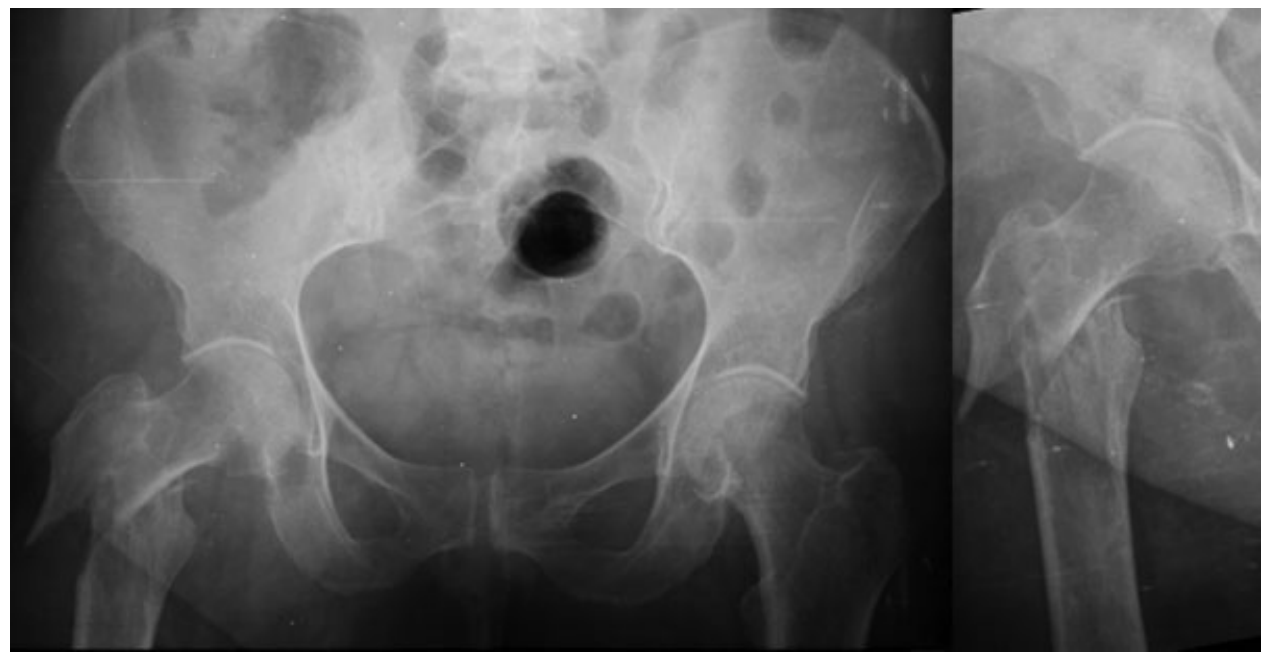

Fig. 4 Radiografias anteroposterior da bacia e perfil coxofemoral; classificação AO 31 A3. 
Qual subgrupo da classificação sofre maior variação na classificação? 0 uso da tomografia computadorizada promove alteração na escolha do tratamento?

Utilizando a classificação AO simplificada, as fraturas instáveis (A3) classificadas por TC apresentaram maior reprodutibilidade do que as classificadas apenas por radiografia simples. ${ }^{30}$

0 estudo de Van Embden D. et al. ${ }^{33}$ esses resultados quando a TC é acrescentada na análise. Neste estudo, as fraturas do tipo A3 apresentaram menor concordância e maior número de modificações de escolha de implantes cirúrgicos quando comparadas ao implante escolhido baseado apenas nas avaliações radiográficas simples ${ }^{33}$ (-Figs. 2 a 4). Vale ressaltar que as fraturas do tipo A3 apresentam as maiores taxas de complicação, como pseudoartrose e falha na fixação em até $32 \%$ dos casos. ${ }^{33}$

Mesmo que as fraturas do tipo A3 sofram as maiores modificações em sua classificação, no trabalho de Van Embden D. et al. ${ }^{33}$ as fraturas dos tipos A1 e A2 também tiveram modificação na escolha dos implantes após a realização da TC em 11 dos 30 casos do estudo. ${ }^{33}$ Além disso, Shen et al, ${ }^{31}$ ao utilizarem a TC, demonstraram que a melhor compreensão da fratura promoveu uma redução estatisticamente significativa do tempo de cirurgia quando optado pela fixação com hastes intramedulares, independentemente da classificação.

Logo, acrescentar o exame de TC para fraturas instáveis é plausível, pois ocasiona uma melhor compreensão do traço de fratura, facilita a escolha adequada do implante e é economicamente menos custoso quando comparado aos custos do aumento do tempo cirúrgico e/ou de complicações. No entanto, a questão é se este exame também não seria benéfico para as fraturas simples e consideradas estáveis devido à possibilidade de imprecisões diagnósticas e, consequentemente, de escolhas equivocadas de implantes e de complicações.

Há influência da especialidade e/ou da experiência dos avaliadores nos valores de reprodutibilidade?

Uma das dificuldades para a avaliação dos artigos é a divergência do tipo e do nível da especialidade dos avaliadores. Dois trabalhos permitiram a comparação entre radiologistas e ortopedistas. Chapman CB. et al. ${ }^{32}$ demonstram reprodutibilidade forte para radiologistas $(0,67)$ e moderada para ortopedistas $(0,57)$ para classificação AO completa e Evans-Jensen. Mihir Thanvi et $\mathrm{al}^{29}$ apresentaram menor reprodutibilidade entre radiologistas em relação aos ortopedistas, tanto para AO simplificada quanto completa através da análise radiográfica, tomográfica, e tomográfica com reconstrução 3D.

Além disso, como exemplificado no trabalho de Isida R. et al., ${ }^{30}$ verificou-se viés na avaliação da reprodutibilidade relacionada à especialidade do avaliador. 0 ortopedista apresentou reprodutibilidade moderada $(0,45)$, e o radiologista excelente $(0,94)$, porém o primeiro avaliou exclusivamente a radiografia e o segundo apenas a TC. De forma que não nos permite concluir que a especialidade influencia na reprodutibilidade.
Outra variável que poderia influenciar na reprodutibilidade é a experiência do avaliador. Neste sentido, encontramse tanto trabalhos nos quais médicos residentes apresentam erros de classificação e menor reprodutibilidade, principalmente em fraturas instáveis, ${ }^{20,33}$ quanto trabalhos nos quais avaliações por médicos menos experientes não comprometeram a qualidade da classificação em relação aos mais experientes. $^{29}$

Van Embden et al ${ }^{33}$ deram suporte a essa última afirmação ao demonstrar que residentes obtiveram reprodutibilidade semelhante tanto aos cirurgiões de trauma quanto a radiologistas. Contudo, Cavaignac E. et al, ${ }^{20}$ mesmo concordando que avaliadores menos experientes não comprometem o estudo, demonstraram reprodutibilidade interobservador excelente $(0,85)$ para avaliadores sênior e forte $(0,79)$ para avaliadores júnior ao acrescentar a TC.

\section{Limitações}

Há uma grande variação no número de avaliadores nos artigos da revisão (entre 2 e 11 indivíduos) e grande variação nas especialidades dos avaliadores: radiologistas, ortopedistas e residentes de ambas as especialidades, configurando diferentes graus de experiência e de conhecimento técnico.

A discrepância do número amostral dos trabalhos também foi fator importante (de 30 até 110 pacientes). Este fator isolado poderia justificar variações dos valores de kappa, uma vez que o intervalo de confiança (IC) para a concordância interobservador depende diretamente do número da amostra e de avaliadores, sendo os mesmos inversamente proporcionais. ${ }^{37}$ Por fim, a utilização de diferentes métodos e a dificuldade de padronização comprometeram a análise dos dados e a interpretação dos resultados.

\section{Considerações Finais}

Há evidências de que há beneficio para o uso da TC sobretudo para fraturas consideradas instáveis. O acréscimo da tomografia rotineiramente como ferramenta para garantir melhor reprodutibilidade (intra- e interobservador), assim como para tomada de decisão no tratamento das fraturas transtrocanterianas ainda permanece controversa. Acreditamos que se faz necessário o desenvolvimento de estudos com melhor nível de evidência e com metodologias equivalentes para elucidar os benefícios da TC como ferramenta para classificação das fraturas transtrocanterianas.

Conflitos de interesses

Os autores declaram não haver conflitos de interesses.

\section{Referências}

1 Müller ME, Nazarian S, Koch P. Classification AO des fractures. Berlin: Springer-Verlag; 1987

2 Pervez H, Parker MJ, Pryor GA, Lutchman L, Chirodian N. Classification of trochanteric fracture of the proximal femur: a study of the reliability of current systems. Injury 2002;33(08): 713-715 
3 Burge R, Dawson-Hughes B, Solomon DH, Wong JB, King A, Tosteson A. Incidence and economic burden of osteoporosisrelated fractures in the United States, 2005-2025. J Bone Miner Res 2007;22(03):465-475

4 Lenich A, Vester H, Nerlich M, Mayr E, Stöckle U, Füchtmeier B. Clinical comparison of the second and third generation of intramedullary devices for trochanteric fractures of the hip-Blade vs screw. Injury 2010;41(12):1292-1296

5 Andruszkow H, Frink M, Frömke C, et al. Tip apex distance, hip screw placement, and neck shaft angle as potential risk factors for cut-out failure of hip screws after surgical treatment of intertrochanteric fractures. Int Orthop 2012;36(11):2347-2354

6 Parker M, Johansen A. Hip fracture. BMJ 2006;333(7557):27-30

7 Hip fracture: management. Guidance and guidelines | NICE [acesso em 9 fevereiro, 2018]. Disponível em: https://www.nice.org.uk/ guidance $/ \operatorname{cg} 124$

8 Tronzo RG. Symposium on fractures of the hip. Special considerations in management. Orthop Clin North Am 1974;5(03):571-583

9 Yang S, Liu Y, Yang T, Zou J, Yang H. Early Clinical Efficacy Comparison Study of Gamma3 Nail, Percutaneous Compression Plate (PCCP) and Femoral Head Replacement (FHR) Treatment on Senile Unstable Intertrochanteric Fractures. J Invest Surg 2018;31(02):130-135

10 Shin YS, Chae JE, Kang TW, Han SB. Prospective randomized study comparing two cephalomedullary nails for elderly intertrochanteric fractures: Zimmer natural nail versus proximal femoral nail antirotation II. Injury 2017;48(07):1550-1557

11 Koval KJ, Oh CK, Egol KA. Does a traction-internal rotation radiograph help to better evaluate fractures of the proximal femur? Bull NYU Hosp Jt Dis 2008;66(02):102-106

12 Palm H, Jacobsen S, Sonne-Holm S, Gebuhr P; Hip Fracture Study Group. Integrity of the lateral femoral wall in intertrochanteric hip fractures: an important predictor of a reoperation. J Bone Joint Surg Am 2007;89(03):470-475

13 Fung W, Jonsson A, Buhren V, Bhandari M. Classifying intertrochanteric fractures of the proximal femur: does experience matter? Med Princ Pract 2007;16(03):198-202

14 Boyd H, Griffin L. Classification and treatment of trochanteric fracture. Arch Surg 1949;58(06):553-566

15 Jensen JS. Classification of trochanteric fractures. Acta Orthop Scand 1980;51(05):803-810

16 Evans EM. The treatment of trochanteric fractures of the femur. J Bone Joint Surg Br 1949;31B(02):190-203

17 Jin WJ, Dai LY, Cui YM, Zhou Q, Jiang LS, Lu H. Reliability of classification systems for intertrochanteric fractures of the proximal femur in experienced orthopaedic surgeons. Injury 2005; 36(07):858-861

18 Schipper IB, Steyerberg EW, Castelein RM, van Vugt AB. Reliability of the AO/ASIF classification for pertrochanteric femoral fractures. Acta Orthop Scand 2001;72(01):36-41

19 Urrutia J, Zamora T, Besa P, Zamora M, Schweitzer D, Klaber I. Inter and intra-observer agreement evaluation of the $\mathrm{AO}$ and the Tronzo classification systems of fractures of the trochanteric area. Injury 2015;46(06):1054-1058

20 Cavaignac E, Lecoq M, Ponsot A, et al. CT scan does not improve the reproducibility of trochanteric fracture classification: a prospective observational study of 53 cases. Orthop Traumatol Surg Res 2013;99(01):46-51
21 van Embden D, Rhemrev SJ, Meylaerts SA, Roukema GR. The comparison of two classifications for trochanteric femur fractures: the AO/ASIF classification and the Jensen classification. Injury 2010;41(04):377-381

22 Zwipp H, Amlang M. [Treatment of fractures of the ankle in the elderly]. Orthopade 2014;43(04):332-338

23 Haapamaki VV, Kiuru MJ, Koskinen SK. Ankle and foot injuries: analysis of MDCT findings. AJR Am J Roentgenol 2004;183(03):615-622

24 Chan PS, Klimkiewicz JJ, Luchetti WT, et al. Impact of CT scan on treatment plan and fracture classification of tibial plateau fractures. J Orthop Trauma 1997;11(07):484-489

25 Mustonen AO, Koskinen SK, Kiuru MJ. Acute knee trauma: analysis of multidetector computed tomography findings and comparison with conventional radiography. Acta Radiol 2005;46(08):866-874

26 Doornberg J, Lindenhovius A, Kloen P, van Dijk CN, Zurakowski D, Ring D. Two and three-dimensional computed tomography for the classification and management of distal humeral fractures. Evaluation of reliability and diagnostic accuracy. J Bone Joint Surg Am 2006;88(08):1795-1801

27 Humphrey CA, Dirschl DR, Ellis TJ. Interobserver reliability of a CT-based fracture classification system. J Orthop Trauma 2005;19 (09):616-622

28 Nakano T. Proposal for understanding trochanteric femoral fractures in the elderly and classification using 3DCT. Orthopaedics (Glendale Calif) 2006;19(05):39-45

29 Mihir Thanvi, Gupta AK, Goyal N, Agarwal R, Sharma C. Reliability of commonly used classification systems of trochanteric fractures of the proximal femur and evaluation of $\mathrm{CT}$ in improving the interobserver agreement. Sch J App Med Sci. 2013;1(06):706-709

30 Isida R, Bariatinsky V, Kern G, Dereudre G, Demondion X, Chantelot $C$. Prospective study of the reproducibility of X-rays and CT scans for assessing trochanteric fracture comminution in the elderly: a series of 110 cases. Eur J Orthop Surg Traumatol 2015;25(07):1165-1170

31 Shen J, Hu F, Zhang L, Tang P, Bi Z. Preoperative classification assessment reliability and influence on the length of intertrochanteric fracture operations. Int Orthop 2013;37(04):681-687

32 Chapman CB, Herrera MF, Binenbaum G, et al. Classification of intertrochanteric fractures with computed tomography: a study of intraobserver and interobserver variability and prognostic value. Am J Orthop 2003;32(09):443-449

33 van Embden D, Scheurkogel MM, Schipper IB, Rhemrev SJ, Meylaerts SA. The value of CT compared to radiographs in the classification and treatment plan of trochanteric fractures. Arch Orthop Trauma Surg 2016;136(08):1091-1097

34 Jüni P, Egger M. PRISMAtic reporting of systematic reviews and meta-analyses. Lancet 2009;374(9697):1221-1223

35 Landis JR, Koch GG. The measurement of observer agreement for categorical data. Biometrics 1977;33(01):159-174

36 Ito R, Obara S, Atsumi T. Radiographic analysis of preoperative and postoperative 3dct images of trochanteric femoral fractures. Showa Univ J Med Sci. 2014;26(04):271-282

37 Rotondi MA, Donner A. A confidence interval approach to sample size estimation for interobserver agreement studies with multiple raters and outcomes. J Clin Epidemiol 2012;65(07):778-784 